\title{
Lymphopenia at 4 Days Postoperatively Is the Most Significant Laboratory Marker for Early Detection of Surgical Site Infection Following Posterior Lumbar Instrumentation Surgery
}

\author{
Eiichiro Iwata ${ }^{1}$, Hideki Shigematsu ${ }^{1}$, Akinori Okuda ${ }^{1}$, Yasuhiko Morimoto ${ }^{1}$, \\ Keisuke Masuda ${ }^{1}$, Hiroshi Nakajima ${ }^{2}$, Munehisa Koizumi ${ }^{3}$, Yasuhito Tanaka ${ }^{1}$ \\ ${ }^{1}$ Department of Orthopedic Surgery, Nara Medical University, Nara, Japan \\ ${ }^{2}$ Department of Orthopedic Surgery, Otemae Hospital, Osaka, Japan \\ ${ }^{3}$ Department of Spine Surgery, Nara Prefecture General Medical Center, Nara, Japan
}

\begin{abstract}
Study Design: Case control study.
Purpose: To identify the most significant laboratory marker for early detection of surgical site infection (SSI) using multiple logistic regression analysis.

Overview of Literature: SSI is a serious complication of spinal instrumentation surgery. Early diagnosis and treatment are crucial.

Methods: We retrospectively reviewed the laboratory data of patients who underwent posterior lumbar instrumentation surgery for degenerative spinal disease from January 2003 to December 2014. Six laboratory markers for early SSI detection were considered: renewed elevation of the white blood cell count, higher at 7 than 4 days postoperatively; renewed elevation of the C-reactive protein (CRP) level, higher at 7 than 4 days postoperatively; CRP level of $>10 \mathrm{mg} / \mathrm{dL}$ at 4 days postoperatively; neutrophil percentage of $>75 \%$ at 4 days postoperatively; lymphocyte percentage of $<10 \%$ at 4 days postoperatively; and lymphocyte count of $<1,000 / \mu \mathrm{L}$ at 4 days postoperatively.

Results: Ninety patients were enrolled; five developed deep SSI. Multivariate regression analysis showed that a lymphocyte count of $<1,000 / \mu \mathrm{L}$ at 4 days postoperatively was the sole significant independent laboratory marker for early detection of SSI ( $p=0.037$; odds ratio, 11.9; 95\% confidence interval, 1.2-122.7).

Conclusions: A lymphocyte count of $<1,000 / \mu \mathrm{L}$ at 4 days postoperatively is the most significant laboratory marker for early detection of SSI.
\end{abstract}

Keywords: Surgical wound infection; Instrumentation; Biomarkers; Lymphopenia; Leukocytes

\section{Introduction}

Surgical site infection (SSI) remains one of the most serious problems after spinal instrumentation surgery [1-3]. SSI may have important long-term consequences that affect final outcomes, including the need for revision surgery,

Received Jan 22, 2016; Revised Apr 23, 2016; Accepted May 10, 2016

Corresponding author: Eiichiro Iwata

Department of Orthopedic Surgery, Nara Medical University, 840 Shijo-cho, Kashiharashi, Nara 634-8522, Japan

Tel: +81-744-29-8873, Fax: +81-744-29-4902, E-mail: iwata@naramed-u.ac.jp 
persistent pain or deformity, additional hospitalization, prolonged recovery time, and considerable expenses [46]. Infection rates after spinal instrumentation surgery reportedly range from $2.2 \%-8.5 \%$ [7-10]. Preventing SSI is important; however, when an infection does occur, early diagnosis and treatment are crucial to avoid aggravation [4,11-13]. A diagnosis of SSI should be made based on indicators of systemic infection, such as body temperature (BT), laboratory data, imaging techniques (e.g., computed tomography, magnetic resonance imaging, and fluorine18-fluorodeoxglucose positron emission tomography) [14-17], and local findings such as tenderness, swelling, redness, and purulent discharge $[11,13]$. However, high BT is not a specific sign of SSI $[11,12,18,19]$, and the above-mentioned imaging techniques are expensive when used as screening tools. Therefore, postoperative laboratory markers are frequently used to screen for SSI because of their objectivity and convenience $[4,11,12,18]$. Several laboratory markers for early detection of SSI have been reported $[4,12]$. The present study aimed to identify the most significant laboratory marker for early detection of SSI using multiple logistic regression analysis.

\section{Materials and Methods}

After receiving approval from the Institutional Review Board of the participating institutions, we retrospectively reviewed the medical records of patients who underwent posterior lumbar instrumentation surgery for degenerative spinal disease from January 2003 to December 2014. The records were examined for the evidence of deep SSI and to collect laboratory data. A diagnosis of SSI was defined using the Centers for Disease Control and Prevention criteria [20]. A diagnosis of deep SSI was made only if the attending surgeon diagnosed deep SSI because of recognizing fever or wound discharge amongst other symptoms, and conducted debridement within 4 weeks. Each patient underwent single or two-segment posterior lumbar instrumentation surgery and laboratory data collection preoperatively and at 4 and 7 days postoperatively. Patients with trauma, tumors, infection, or chronic inflammatory disease (e.g., rheumatoid arthritis), those undergoing dialysis, those with a preoperative C-reactive protein (CRP) level of $>2 \mathrm{mg} / \mathrm{dL}$, and those with a history of surgery to the lumbar spine were excluded. The enrolled patients were categorized into two groups: an SSI group and a non-SSI group. In total, 90 patients' data were evaluated in the study. Of these patients, five developed deep SSI. We collected laboratory data regarding the CRP level, white blood cell (WBC) count, and WBC differential (neutrophil and lymphocyte percentages) preoperatively and at 4 and 7 days postoperatively. The CRP level was measured using the latex agglutination method, and an automatic cell counter determined the WBC count. Neutrophil and lymphocyte counts were calculated from the WBC count and WBC differential. The operating time and intraoperative blood loss volume were also measured.

Six laboratory markers for early detection of SSI were reviewed $[11,12,18,19]$ : renewed elevation of the WBC count, higher at 7 than at 4 days postoperatively; renewed elevation of the CRP level, higher at 7 than at 4 days postoperatively; CRP level of $>10 \mathrm{mg} / \mathrm{dL}$ at 4 days postoperatively; neutrophil percentage of $>75 \%$ at 4 days postoperatively; lymphocyte percentage of $<10 \%$ at 4 days postoperatively; and lymphocyte count of $<1,000 / \mu \mathrm{L}$ at 4 days postoperatively. Univariate analysis was performed to identify the associations between each laboratory marker and the presence of SSI. Quantitative data were analyzed with the Mann-Whitney U-test and Student's $t$-test. Qualitative data were analyzed with Fisher's exact probability test. To identify independent predictive laboratory markers for early detection of SSI, multivariate logistic regression analysis was performed using variables with a $p$-value of $<0.20$ in the univariate analysis by stepwise forwardselection. All statistical analyses were carried out using SPSS ver. 22.0 (IBM Corp., Armonk, NY, USA). A p-value of $<0.05$ was considered statistically significant.

\section{Results}

Ninety patients' data were evaluated in the present study. Five patients who developed deep SSI were assigned to the SSI group ( 1 man, 4 women), and the remaining 85 patients were assigned to the non-SSI group (31 men, 54 women). The median ages at surgery in the SSI and nonSSI groups were 73.0 and 68.5 years, respectively. The mean operating times in the SSI and non-SSI groups were 170 minutes (range, 143-315 minutes) and 243 minutes (range, 80-410 minutes), respectively, and the mean intraoperative blood loss volumes were $305 \mathrm{~mL}$ (range, $100-600 \mathrm{~mL}$ ) and $345 \mathrm{~mL}$ (range, 20-2,440 mL), respectively. The mean \pm standard deviation of fused segments in the SSI and non-SSI groups was $1.4 \pm 0.5$ and $1.2 \pm 0.4$, respectively. There were no significant differences in age, 
Table 1. Patients' demographic data

\begin{tabular}{lccc} 
Variable & $\begin{array}{c}\text { SSI group } \\
(\mathrm{n}=5)\end{array}$ & $\begin{array}{c}\text { Non-SSI group } \\
(\mathrm{n}=85)\end{array}$ & $p$-value \\
Age (yr) & $73.0(57-78)$ & $68.5(33-84)$ & 0.277 \\
Sex (male:female) & $1: 4$ & $31: 54$ & 0.413 \\
Operating time (min) & $170(143-315)$ & $243(80-410)$ & 0.262 \\
Blood loss (mL) & $305(100-600)$ & $345(20-2,440)$ & 0.906 \\
No. of fused segments & $1.4 \pm 0.5$ & $1.2 \pm 0.4$ & 0.185 \\
\hline
\end{tabular}

Values are presented as median (range) or mean \pm standard deviation unless otherwise indicated.

SSI, surgical site infection.

Table 2. Univariate analysis between SSI and non-SSI groups

\begin{tabular}{|c|c|c|c|}
\hline Variable & $\begin{array}{l}\text { SSI groups } \\
\qquad(n=5)\end{array}$ & $\begin{array}{l}\text { Non-SSI groups } \\
(n=85)\end{array}$ & $p$-value \\
\hline Renewed elevation of white blood cell count, 7 (>4 days postoperatively) & 1 & 18 & 0.690 \\
\hline Renewed elevation of $\mathrm{C}$-reactive protein level, 7 (>4 days postoperatively) & 1 & 13 & 0.603 \\
\hline C-reactive protein level of $>10 \mathrm{mg} / \mathrm{dL}$ at 4 days postoperatively & 2 & 2 & $0.023^{\mathrm{a})}$ \\
\hline Neutrophil percentage of $>75 \%$ at 4 days postoperatively & 2 & 11 & 0.166 \\
\hline Lymphocyte percentage of $<10 \%$ at 4 days postoperatively & 0 & 3 & 0.832 \\
\hline Lymphocyte count of $<1,000 / \mu \mathrm{L}$ at 4 days postoperatively & 4 & 16 & $0.010^{\mathrm{a})}$ \\
\hline
\end{tabular}

Positive patient's number.

SSI, surgical site infection.

a) Statistically significant $(p<0.05)$.

Table 3. Multivariate analysis of predictive laboratory markers for early detection of surgical site infection

\begin{tabular}{lccr} 
& $p$-value & Odds ratio & $95 \%$ Confidence interval \\
Lymphocyte count of $<1,000 / \mu \mathrm{L}$ at 4 days postoperatively & 0.037 & 11.9 & $1.2-122.7$ \\
\hline
\end{tabular}

sex, operating time, intraoperative blood loss, or the number of fused segments between the two groups (Table 1). Univariate analysis revealed a statistically significant difference in the CRP level of $>10 \mathrm{mg} / \mathrm{dL}$ at 4 days postoperatively and the lymphocyte count of $<1,000 / \mu \mathrm{L}$ at 4 days postoperatively between the two groups ( $p=0.023$ and $p=0.010$, respectively) (Table 2 ). On multiple logistic regression, variables with a $p$-value of $<0.20$ on univariate analysis (CRP level of $>10 \mathrm{mg} / \mathrm{dL}$ at 4 days postoperatively, neutrophil percentage of $>75 \%$ at 4 days postoperatively, and lymphocyte count of $<1,000 / \mu \mathrm{L}$ at 4 days postoperatively) were analyzed as dependent variables. A lymphocyte count of $<1,000 / \mu \mathrm{L}$ at 4 days postoperatively was the only significant independent laboratory marker for early detection of SSI ( $p=0.037$; odds ratio, $11.9 ; 95 \%$ confidence interval, 1.2-122.7) (Table 3).

\section{Discussion}

The objective of SSI treatment after spinal instrumentation surgery is not only to resolve infection but also to maintain spinal stability. Ishii et al. [21] reported that patients who developed SSI and were able to retain their implants were suspected and diagnosed at an early stage. They also found that early diagnosis at a low intensity of infection may reduce the necessity of implant removal. Diagnosis of SSI may be made based on signs of systemic infection, such as BT, laboratory markers, and some imaging techniques, in combination with local findings, such as tenderness, swelling, redness, and purulent discharge $[6,13]$. However, some authors have reported high BT as not a SSI-specific sign $[11,12,18,19]$, and imaging techniques are expensive when used as a screening tool. Therefore, 
it is impossible to use these tests in all cases. Postoperative laboratory markers are frequently used as a screening test for diagnosis of SSI because of their objectivity and convenience $[4,11,12,18]$. The most widely implemented laboratory markers are the CRP level and WBC count, which can easily be measured at most institutions. In the present study, we reviewed six laboratory markers related to the CRP level and WBC count for early detection of SSI as previously reported $[11,12,18,19]$. Several authors have reported that the CRP level is the most sensitive inflammatory marker $[18,22]$. CRP is induced by interleukin- 6 and synthesized by hepatocytes; its level thus reflects the degree of inflammatory response and surgical injury [23]. Larsson et al. [22] were the first to report changes in the CRP level after posterior lumbar instrumentation surgery. They found that the level tended to peak after postoperative day 3. However, Takahashi et al. [18] reported that the postoperative CRP level peaked on day 2, and Aono et al. [12] reported that it peaked on day 4 . With the peak in the postoperative CRP level ranging from day 2 to 4 , all four groups agreed that renewed elevation of the CRP level or an increase in the CRP level after the peak day suggests the possibility of SSI $[11,12,23]$. Takahashi and Collegues $[11,18]$ reported that the WBC count and WBC differential are useful for early detection of surgical wound infection following spinal instrumentation surgery. Furthermore, changes in the WBC count, particularly the neutrophil count, serves as a useful marker of postoperative progress over time [18]. Takahashi et al. [18] also reported that another increase in the neutrophil and WBC counts several days after surgery was one of the most important signs of bacterial infection. They concluded that a renewed elevation of the postoperative WBC and neutrophil counts after days 4 to 7 and a postoperative neutrophil percentage of $>75 \%$ after day 4 may be critical signs of infection $[11,18]$. Conversely, lymphocytes, which are involved in nonspecific biophylaxis, often decrease after invasion, regardless of noninfectious or infectious invasion. They reported that in patients with infection, the percentage and number of lymphocytes significantly decreased on day 4 . This represents immune depression and indicates the possibility of predominance of anti-inflammatory cytokines and attendant compensatory anti-inflammatory response syndrome [24]. Patients having this condition are more susceptible to and often develop postoperative infection. They concluded that postoperative lymphopenia of $<10 \%$ or $<1,000 / \mu \mathrm{L}$ after
4 days indicates a possible surgical wound infection $[11,18]$. With reference to these reports, we identified the most significant laboratory marker for early detection of SSI using multiple logistic regression analysis. As a result, a lymphocyte count of $<1,000 / \mu \mathrm{L}$ at 4 days postoperatively was the only significant independent laboratory marker for early detection of SSI.

Our study has several limitations. First, as it was a retrospective study, there may have been an inherent bias associated with patient selection and missing patient information. Patients who did not meet SSI criteria were placed in a non-SSI group, which may reflect a significant underestimation of the actual number of SSI cases. Another limitation is the possibility that a type 2 error may have occurred because of the relatively small number of SSI cases. Herein, only a lymphocyte count of $<1,000 / \mu \mathrm{L}$ at 4 days postoperatively was considered statistically significant in the multiple logistic regression analysis; however, if a higher number of SSI cases had been evaluated, another statistically significant index may have been identified. A prospective study and larger cohort may address these problems.

\section{Conclusions}

The present study indicates that a lymphocyte count of $<1,000 / \mu \mathrm{L}$ at 4 days postoperatively is the most significant laboratory marker for early detection of SSI.

\section{Conflict of Interest}

No potential conflict of interest relevant to this article was reported.

\section{References}

1. Olsen MA, Nepple JJ, Riew KD, et al. Risk factors for surgical site infection following orthopaedic spinal operations. J Bone Joint Surg Am 2008;90:62-9.

2. Pull ter Gunne AF, Cohen DB. Incidence, prevalence, and analysis of risk factors for surgical site infection following adult spinal surgery. Spine (Phila Pa 1976) 2009;34:1422-8.

3. Fang A, Hu SS, Endres N, Bradford DS. Risk factors for infection after spinal surgery. Spine (Phila $\mathrm{Pa}$ 1976) 2005;30:1460-5.

4. Mok JM, Guillaume TJ, Talu U, et al. Clinical out- 
come of deep wound infection after instrumented posterior spinal fusion: a matched cohort analysis. Spine (Phila Pa 1976) 2009;34:578-83.

5. Gerometta A, Rodriguez Olaverri JC, Bitan F. Infections in spinal instrumentation. Int Orthop 2012;36: 457-64.

6. Kurtz SM, Lau E, Ong KL, et al. Infection risk for primary and revision instrumented lumbar spine fusion in the medicare population. J Neurosurg Spine 2012; 17:342-7.

7. Collins I, Wilson-MacDonald J, Chami G, et al. The diagnosis and management of infection following instrumented spinal fusion. Eur Spine J 2008;17:44550.

8. Ho C, Skaggs DL, Weiss JM, Tolo VT. Management of infection after instrumented posterior spine fusion in pediatric scoliosis. Spine (Phila Pa 1976) 2007;32: 2739-44.

9. Pull ter Gunne AF, Mohamed AS, Skolasky RL, van Laarhoven CJ, Cohen DB. The presentation, incidence, etiology, and treatment of surgical site infections after spinal surgery. Spine (Phila Pa 1976) 2010; 35:1323-8.

10. Schimmel JJ, Horsting PP, de Kleuver M, Wonders G, van Limbeek J. Risk factors for deep surgical site infections after spinal fusion. Eur Spine J 2010;19:17119.

11. Takahashi J, Shono Y, Hirabayashi H, et al. Usefulness of white blood cell differential for early diagnosis of surgical wound infection following spinal instrumentation surgery. Spine (Phila Pa 1976) 2006;31:1020-5.

12. Aono H, Ohwada T, Kaneko N, Fuji T, Iwasaki M. The post-operative changes in the level of inflammatory markers after posterior lumbar interbody fusion. J Bone Joint Surg Br 2007;89:1478-81.

13. Mangram AJ, Horan TC, Pearson ML, Silver LC, Jarvis WR. Guideline for prevention of surgical site infection, 1999. Hospital Infection Control Practices Advisory Committee. Infect Control Hosp Epidemiol 1999;20:250-78.
14. Tsiodras S, Falagas ME. Clinical assessment and medical treatment of spine infections. Clin Orthop Relat Res 2006;444:38-50.

15. An HS, Seldomridge JA. Spinal infections: diagnostic tests and imaging studies. Clin Orthop Relat Res 2006;444:27-33.

16. Gemmel F, Dumarey N, Palestro CJ. Radionuclide imaging of spinal infections. Eur J Nucl Med Mol Imaging 2006;33:1226-37.

17. Walker RC, Jones-Jackson LB, Martin W, Habibian MR, Delbeke D. New imaging tools for the diagnosis of infection. Future Microbiol 2007;2:527-54.

18. Takahashi J, Ebara S, Kamimura M, et al. Early-phase enhanced inflammatory reaction after spinal instrumentation surgery. Spine (Phila Pa 1976) 2001;26: 1698-704.

19. Deguchi M, Shinjo R, Yoshioka Y, Seki H. The usefulness of serum amyloid A as a postoperative inflammatory marker after posterior lumbar interbody fusion. J Bone Joint Surg Br 2010;92:555-9.

20. Horan TC, Gaynes RP, Martone WJ, Jarvis WR, Emori TG. CDC definitions of nosocomial surgical site infections, 1992: a modification of CDC definitions of surgical wound infections. Infect Control Hosp Epidemiol 1992;13:606-8.

21. Ishii M, Iwasaki M, Ohwada T, et al. Postoperative deep surgical-site infection after instrumented spinal surgery: a multicenter study. Global Spine J 2013;3: 95-102.

22. Larsson S, Thelander U, Friberg S. C-reactive protein (CRP) levels after elective orthopedic surgery. Clin Orthop Relat Res 1992;(275):237-42.

23. Ganter U, Arcone R, Toniatti C, Morrone G, Ciliberto G. Dual control of C-reactive protein gene expression by interleukin-1 and interleukin-6. EMBO J 1989;8:3773-9.

24. Takahashi J, Ebara S, Kamimura M, et al. Pro-inflammatory and anti-inflammatory cytokine increases after spinal instrumentation surgery. J Spinal Disord Tech 2002;15:294-300. 